\title{
Werkzeugkasten für Ärztinnen und Ärzte
}

\author{
Stefan Ritler \\ Vizedirektor Bundesamt für Sozialversicherungen BSV, Leiter Geschäftsfeld IV
}

\begin{abstract}
Die adäquate ärztliche Behandlung ist ein wichtiger Faktor für eine erfolgreiche sozial-berufliche Eingliederung von kranken Personen. Sie muss aber gut auf die beruflichen Massnahmen der Invalidenversicherung (IV) abgestimmt sein. Dies kann nur erreicht werden, wenn der behandelnde Arzt und die Mitarbeitenden der IV-Stelle und der regionalärztliche Dienst der IV eng miteinander zusammenarbeiten.
\end{abstract}

Für eine erkrankte Person, die noch einen Arbeits- oder Ausbildungsplatz hat, ist schnelles Handeln besonders wichtig. Der behandelnde Arzt steht hier an vorderster Front. Er kann am besten beurteilen, ob und in welchem Masse sein Patient noch leistungsfähig ist und wo allenfalls bei der Eingliederung Leistungsdefizite zu erwarten sind. Ist er unsicher bei der Festlegung der Arbeitsunfähigkeit, so nimmt er am besten auch unabhängig von der IV und in Absprache mit seinem Patienten mit den Arbeitgebenden oder Ausbildenden Kontakt auf. Dies bringt den Vorteil, dass der behandelnde Arzt vor dem Verfassen eines Arztzeugnisses die Anforderungen am Arbeitsplatz seines Patienten näher kennt. Zur besseren Beurteilung der Arbeitsfähigkeit haben die Ostschweizer Ärzte-

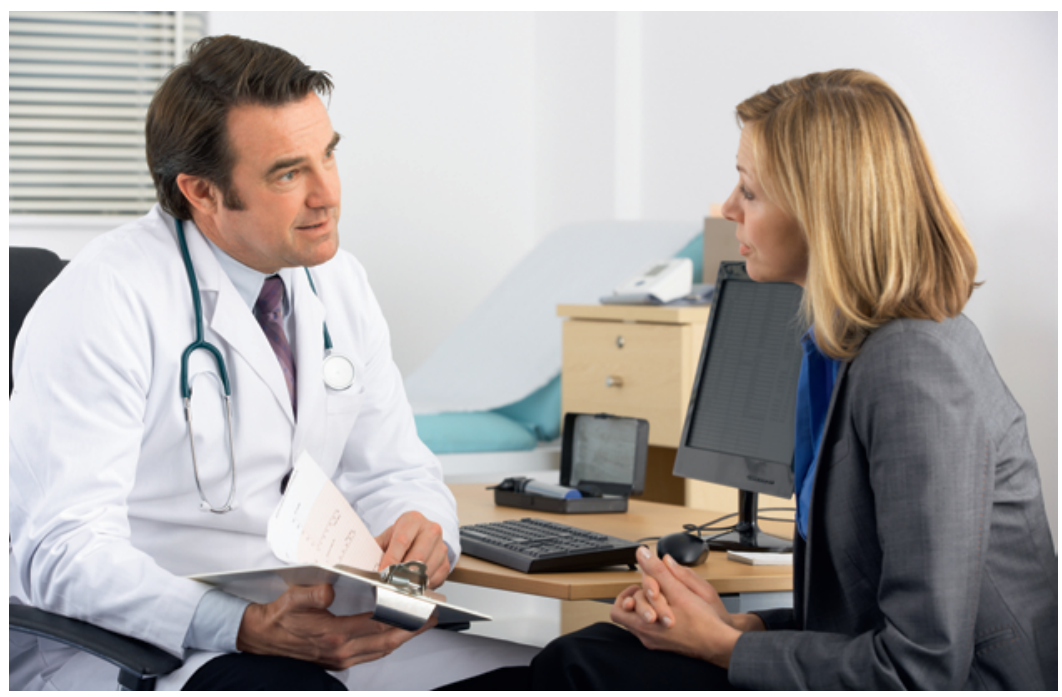

Unabdingbar für eine erfolgreiche berufliche und soziale Wiedereingliederung einer erkrankten Person: der intensive Austausch zwischen behandelndem Arzt und Mitarbeitenden der IV-Stelle (Symbolbild). gesellschaft und die Arbeitgeberverbände im Kanton St. Gallen ein erweitertes Arztzeugnis und einen vom Arbeitgeber auszufüllenden Arbeitsplatzbeschrieb entwickelt (Wiedersheim P., 2014). Dieses erweiterte Arztzeugnis ist auch in die Vorlagen der Swiss Insurance

Das Bildungszentrum der IV bietet neu einen kostenlosen Kurs für Spital- und andere Ärzte an. Er gibt einen Überblick über das Sozialversicherungssystem in der Schweiz und das Funktionieren der IV.

Medicine eingeflossen (Link: www.swiss-insurancemedicine.ch). Es wird aktuell vom Verein Compasso, der vom Schweizerischen Arbeitgeberverband präsidiert wird, in Zusammenarbeit mit allen relevanten Akteuren weiterentwickelt. Im 2018 wird dieses neue Arztzeugnis veröffentlicht werden. Die Informationsplattform www.compasso.ch ist auch für die behandelnden Ärzte von Interesse.

\section{Der behandelnde Arzt ist ein zentraler Partner der IV}

Der behandelnde Arzt und die IV-Stelle haben ein gemeinsames Interesse daran, dass die betroffene Person trotz krankheitsbedingter Abwesenheit ihren Arbeitsplatz behalten oder in einer angepassten Tätigkeit eingegliedert werden kann. Wenn eine Person in ihrer Arbeitsfähigkeit eingeschränkt ist, ist es am besten, wenn der behandelnde Arzt nach Absprache mit der betroffenen Person sofort mit der zuständigen IV-Stelle Kontakt aufnimmt. Die IV-Stelle bietet dann den Betroffenen, wenn immer möglich mit dem behandelnden Arzt 
und/oder dem Arbeitgeber zusammen, zu einem Erstgespräch auf und klärt mit ihnen gemeinsam die medizinische und die sozial-berufliche Situation des Betroffenen. Je nach Ergebnis des Erstgespräches erfolgt als nächster Schritt entweder eine IV-Anmeldung oder die IV-Stelle weist den Betroffenen der Sozialberatung oder einer anderen Stelle zu, die ihn besser unterstützen kann. Wenn es zu einer IV-Anmeldung kommt, holen die IV-Stellen Informationen beim Krankenversicherer, beim Krankentaggeldversicherer, bei Spitälern und Arbeitgebern ein. Das Zurückgreifen auf bestehende Unterlagen und der direkte Kontakt mit dem behandelnden Arzt können dazu beitragen, dass die IVStelle keinen oder nur einen auf die noch offenen Fragen beschränkten Bericht vom behandelnden Arzt benötigt. Dazu steht ab Anfang 2018 ein neues, modulartig aufgebautes IV-Arztformular zur Verfügung, das es den IV-Stellen ermöglicht, nur die noch notwendigen Fragen an den Arzt zu richten.

Der behandelnde Arzt kann seine Dienstleistungen für die IV, auch das Verfassen eines Arztberichts oder die Teilnahme an Gesprächen, der IV in Rechnung stellen. Wie die IV-Stellen funktionieren, wie ein Eingliederungsprozess bei der IV abläuft, wie er mit der IV-Stelle abrechnet und vieles mehr findet der Arzt auf der von der IV und der FMH gemeinsam getragenen Ärzteplattform www.iv-pro-medico.ch.

\section{Gegenseitiger Informationsaustausch und Bildungsangebote der IV}

Für die erfolgreiche Eingliederung einer erkrankten Person ist es wichtig, dass auch die medizinische Behandlung und die beruflichen Eingliederungsbemühungen wechselseitig aufeinander abgestimmt werden. Konkret bedeutet dies, dass die Zusammenarbeit zwischen der IV-Stelle und dem behandelnden Arzt stets darauf ausgerichtet sein muss, für die betroffene Person eine gute, aber auch einfache und zweckmässige Leistung zu erbringen. Damit der behandelnde Arzt die medi- zinischen Leistungen auf den Eingliederungsprozess ausrichten kann, braucht er auch Informationen zur aktuellen Situation der betroffenen Person vonseiten der IV. Dies war bisher nicht möglich. Die Weiterentwicklung der IV schlägt vor, dass die Mitarbeitenden der IV-Stellen auch Informationen an die behandelnden Ärzte abgeben können. Damit soll der schon lange von beiden Seiten gewünschte gegenseitige unkomplizierte Informationsfluss zwischen der IV und den behandelnden Ärzten möglich werden. Siehe dazu den Artikel «Weiterentwicklung der IV - die wichtigsten Massnahmen im Überblick» in dieser Ausgabe.

Das Bildungszentrum der IV bietet neu einen kostenlosen Kurs für Spital- und andere Ärzte an. Er gibt einen Überblick über das Sozialversicherungssystem in der Schweiz und das Funktionieren der IV. Ein wichtiges Thema ist auch der Stellenwert der Arztberichte und wie diese Berichte sowie die Beratungsleistungen der behandelnden Ärzte mit der IV korrekt abgerechnet werden. Weiter soll dieser Kurs den behandelnden Ärzten mehr Sicherheit geben bei der Beurteilung der Arbeits(un)fähigkeit ihrer Patienten. Weitere Kurse sind im Jahresprogramm des Bildungszentrums unter www.bziv.ch aufgeführt. Eine Anmeldung ist erforderlich und erfolgt ebenfalls über diese Homepage.

Die Massnahmen im Rahmen der Weiterentwicklung der IV sowie die Informationen auf www.iv-promedico.ch sollen dazu dienen, den Vorwurf einer «Black Box IV» zu entkräften, die Zusammenarbeit zwischen der IV und den behandelnden Ärzten zu vereinfachen und zu stärken.

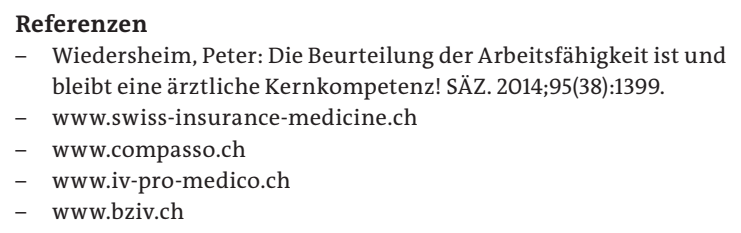

Bildnachweis

(c) Monkeybusinessimages | Dreamstime.com 